Bol. Soc. Bot. México 49:31·39(1989)

\title{
El papel del microclima en la fisiología ecológica vegetal
}

\author{
VICTOR L. BARRADAS ${ }^{1}$
}

\begin{abstract}
RESUMEN. Los avances en el desarrollo de instrumentos de medición más eficientes y precisos han permitido que la micrometereología aporte información de gran valor para la interpretación de los procesos ecofisiológicos de las plantas. En este trabajo se presentan algunos ejemplos concretos de estas aplicaciones en el estudio de la economía del agua y otros procesos tal como tienen lugar en el medio natural.
\end{abstract}

ABSTRACT. The development of more efficient and accurate instruments to measure the microclimatic components have allowed the researches to get environmental information of great value for research on plant physiological ecology. In this paper some examples of applications in the study of water economy and other plant processes are presented. Examples from an area of natural vegetation are given.

\section{INTRODUCCION}

La capa atmosférica en la vecindad de las plantas, es de primera importancia al organismo, ya que estas responden con gran sensibilidad a la forma con que cambian los elementos del tiempo atmosférico y el clima. Aunque el clima global tenga ingerencia en los fenómenos atmosféricos locales, el ambiente cambia muy frecuentemente en la biosfera debido a la interacción de los procesos meteorológicos locales, la topografía y la vegetación.

Uno de los factores más importantes en el clima local o microclima es la topografía, ya que determina el grado en que una superficie se encuentra expuesta a las condiciones del tiempo atmosférico y crea ciertas condiciones, que influencian en el movimiento de masas de aire locales. La vegetación también interactúa con estas condiciones locales afectando las concentraciones de bióxido de carbono, de vapor de agua y de calor en

1

Centro de Ecología.Universidad Nacional Autónoma de México, Apdo. Postal 70-275, Delegación Coyoacán, 04510, México, D. F.

Barradas VL. 1989. El papel del microclima en la fisiología ecológica vegetal. Boletín de la Sociedad Botánica de México 49: 31-39. 
procesos de intercambios gaseosos. De todo esto resulta un intercambio energético responsable de las condiciones microclimáticas que existen en una región.

Algunas variables fisiológicas responden con suma sensibilidad a los microhabitats generados por las condiciones micrometeorológicas tales como la transpiración, la que depende de la apertura estomática (Landsberg, 1984; Jarvis, 1981; Gates, 1980). La transpiración permite reducir la temperatura foliar (Gates, 1980) y protege muchas otras funciones de la planta.

En este trabajo se presentan datos que describen las variaciones de la temperatura foliar y de la transpiración de distintas especies silvestres y cultivadas in situ.

\section{EL MICROCLIMA Y LA VEGETACIÓN}

a) Gradientes verticales del microclima en condiciones naturales.

Los gradientes verticales varían en magnitud entre diferentes sitios pero generalmente son más pronunciados cerca del mediodia en la estación de sequía.

En la Fig. 1 se muestra el comportamiento vertical de la radiación solar, temperatura y humedad del aire e intensidad del viento, en una selva baja caducifolia de la costa de Jalisco. El gradiente más pronunciado en este dosel vegetal es la radiación solar que va de aproximadamente $900 \mathrm{a} 10 \mathrm{w} \mathrm{m}^{-2}$, de 24 a $3 \mathrm{~m}$ de altura, siendo el cambio más drástico de $\operatorname{los} 24$ a los $18 \mathrm{~m}$ de altura.

El viento es generalmente más intenso por encima del dosel vegetal $(5 \mathrm{~m} / \mathrm{s})$, que en su interior; y por debajo de los $12 \mathrm{~m}$ de altura su velocidad es nula.

La temperatura del aire es el elemento que se comporta casi como una constante, con diferencias de apenas $1^{0} \mathrm{C}$. Por otro lado, la humedad tiene variaciones drásticas hacia los 9 y los $21 \mathrm{~m}$ de altura; teniendo sus mayores valores a los 0,15 y $24 \mathrm{~m}$ de altura con 58.557 y $58 \%$ de humedad relativa, mientras que se presentan dos mínimos de 56 y $54 \%$ a los 9 y $21 \mathrm{~m}$ de altura.

b) El microclima en sistemas cultivados a diferentes régimenes de radiación solar.

En la Fig. 2 se muestra el comportamiento de la temperatura máxima y mínima del aire (Tmax y Tmin, respectivamente) y el déficit de presión de vapor (dpv) en dos sistemas de café (Coffea arabica L.) cultivados a sombra y a pleno sol. Las temperaturas máximas del aire (Fig. 2a.) muestran un comportamiento similar en los dos sitios, pero sus magnitudes son muy diferentes. Tmax es más alta en la plantación a pleno sol a lo largo del año (promedio $5.4^{\circ} \mathrm{C}$ ). En el mes más caliente (mayo), Tmax alcanzó los 33.7 ${ }^{0} \mathrm{C}$ en el cultivo a pleno sol y $29.5^{\circ} \mathrm{C}$ en la plantación a sombra; mientras que en enero Tmax en la plantación a sol alcanzó los $22.4^{0} \mathrm{C}$ y a sombra los $17.9^{\circ} \mathrm{C}$, indicando una diferencia de $4.5^{\circ} \mathrm{C}$. Sin embargo, la distribución de Tmin es menos contrastante que la de Tmax, ya que no existen diferencias tan grandes como las que hay en Tmax.

El déficit de presión de vapor se da en la Fig. 2b. La distribución del déficit de presión de vapor a las 1500 hora local (DPV15) tiene su máximo y mínimo valor en abril y julio, respectivamente. La depresión del DPV15 que se observa en julio, proba- 

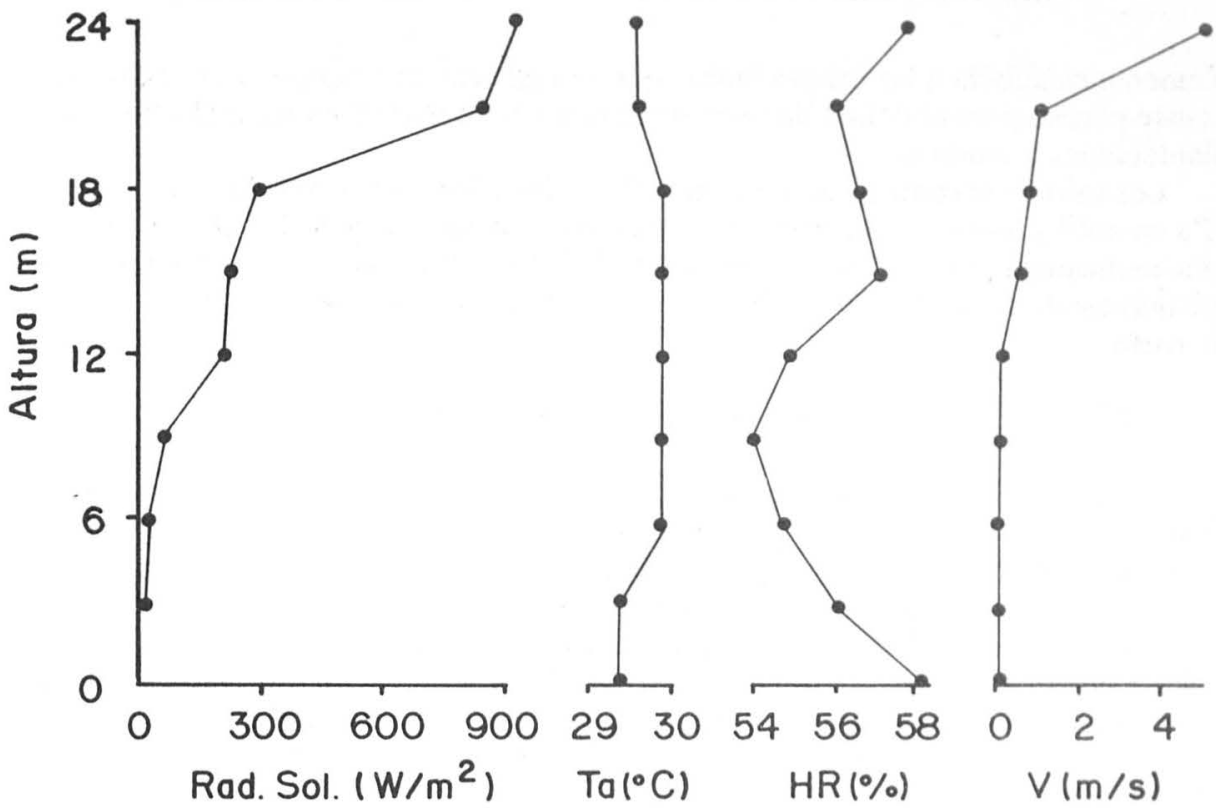

Fig. 1. Gradientes verticales de radiación solar (Rad. Sol.), temperatura del aire (Ta), humedad relativa (HR) y viento (V). en una selva baja caducifollia de la costa de Jalisco.
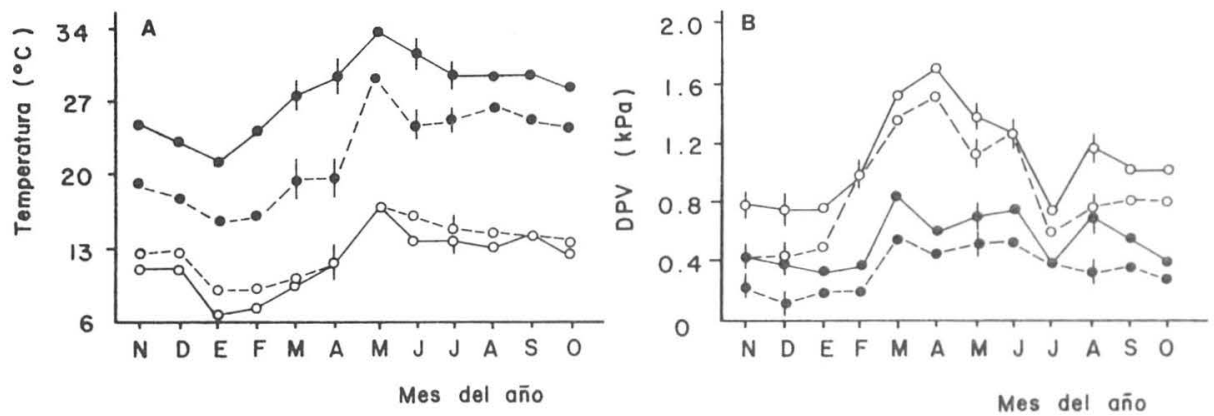

Fig. 2. Comportamiento de la temperatura máxima (símbolos cerrados) y mínima (símbolos abiertos) (a) y el déficit y presión de vapor (dpv) a las 0900 (símbolos cerrados) y a las $1500 \mathrm{hl}$ (símbolos abiertos) (b) en dos plantaciones de café (Coffea arabica L) cultivados a sombra (línea discontínua) y a pleno sol (línea contínua). (Según Barradas y Fanjul, 1986). 
blemente es debida a las fuertes lluvias que se registran en esa época, sin embargo, no es este el caso para el déficit de presión de vapor a las 0900 hora local (DPV09) en las plantaciones a sombra.

Los valores máximos y mínimos de DPV a las 1500 y a las 0900 fueron de 1.7 y 0.7 $\mathrm{kPa}$ en abril y junio, respectivamente, en el sitio a pleno sol, y de $1.5 \mathrm{kPa}$ en abril y 0.4 $\mathrm{kPa}$ en diciembre en el sitio a sombra a las 1500. El comportamiento de DPV a las 0900 fué más estable que el DPV15 a lo largo del año, debido probablemente a la presencia de rocío.

c) El efecto de los rayos de luz en la temperatura foliar.

Como la estructura de un dosel vegetal no es contínua, aparecen lo que se denominan "rayos de luz" que contribuyen con una porción significativa de radiación fotosinteticamente activa disponible para la fotosíntesis, pero que provocan un calentamiento en las hojas de hasta $6^{0} \mathrm{C}$ mayor que el aire.

En la Fig. 3 se muestra el efecto producido de un rayo de luz en la temperatura foliar. El rayo de luz tuvo una duración de cerca de 70 minutos; sin embargo, la nubosidad interfirió en estas mediciones, y por ello, la radiación registrada presenta discontinuidades en su intensidad, pero que interfieren en ambas temperaturas foliares (hoja a la sombra y al sol), teniendo un mayor incremento la temperatura de la hoja expuesta al rayo de luz. Las temperaturas de esta hoja llegan a alcanzar cerca de los $36^{\circ} \mathrm{C}$ y mínimos de $25^{\circ} \mathrm{C}$, mientras que la hoja a la sombra su temperatura varía muy poco de cerca de los 24 a los $28^{0} \mathrm{C}$.

\section{MICROCLIMA Y EVAPOTRANSPIRACIÓN}

a) Transpiración foliar.

La transpiración (TRP) de un individuo se da como la suma de la transpiración de todas sus hojas. Esta se puede determinar por medio de modelos biofísicos como el del balance de energía donde entran en juego todos los parámetros picoambientales, dado a continuación:

$$
R n=((p C p / j)(E s h-E a) /(R s+R a))+p C p((T h+T a) / R h)
$$

donde Esh es la presión de vapor de saturación a la temperatura de la hoja, Ea es la presión de vapor del aire, $\mathrm{Rn}$ es la radiación neta o disponible, $\mathrm{Ra}$ es la resistencia aerodinámica al transporte de vapor de agua, Rs es la resistencia estomática del individuo, $\mathrm{Rh}$ es la resistencia aerodinámica a la transferencia de calor, Th y Ta son la temperatura de la hoja y del aire, respectivamente; L es el calor latente de vaporización y p, Cp y j son constantes. Estrictamente Ra y Rh son diferentes, pero para múltiples propósitos se toman como iguales.

La aplicación de este modelo biofísico determina que la temperatura foliar se incrementa con el aumento de la radiación solar y el decremento de la humedad del aire 


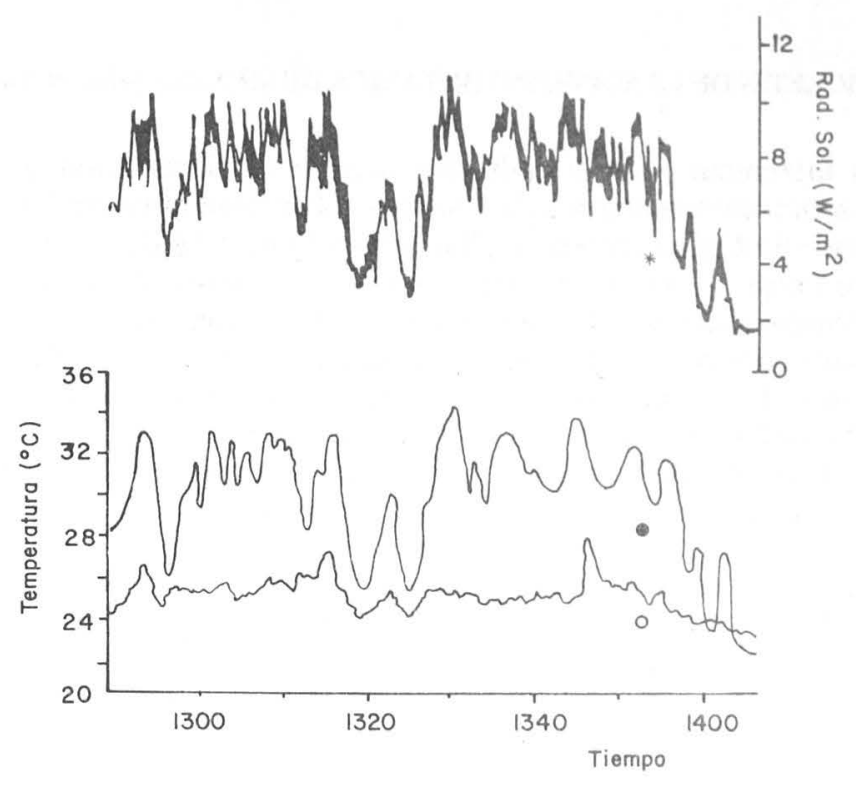

Fig. 3. Efecto producido por un rayo de luz en la temperatura foliar de café (Coffea arabica L.). Radiación solar $\left({ }^{*}\right)$, temperatura de una hoja expuesta a un rayo de luz (0) y temperatura de una hoja a la sombra (0) (Según Barradas y Fanjul, 1983).
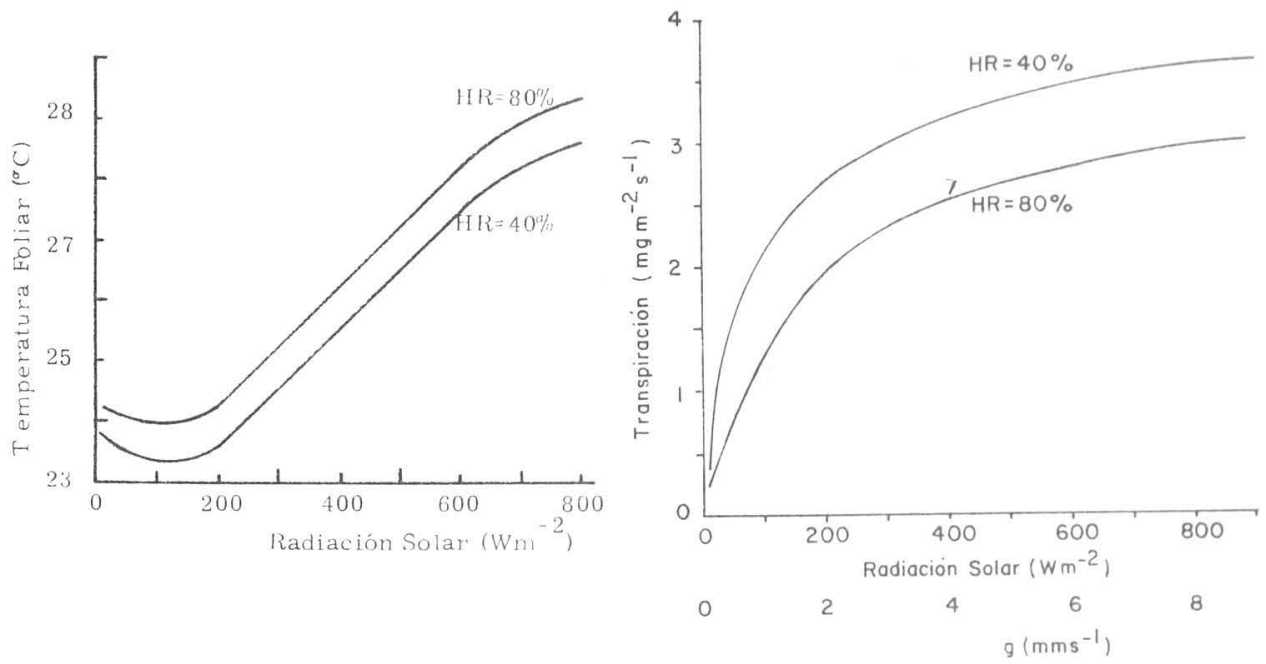

Fig. 4. Temperatura foliar en función de la radiación solar y la humedad relativa.

Fig. 5. Transpiración en función de la radiación solar y la humedad relativa. 
(Fig. 4). Los incrementos de la radiación solar o la disminución de la humedad, ocasionan un sobrecalentamiento de la hoja, este sobrecalentamiento llega a ser de hasta $6^{0} \mathrm{C}$ para el caso de Coffea arabica L (Barradas y Fanjul, 1983). Un incremento de la radiación solar causa un incremento de la transpiración, pero al incrementar la humedad del aire, la transpiración se decrementa, teniendo un efecto contrario a la radiación. Como resultante, se tiene que la velocidad de la transpiración aumenta con el aumento de la radiación y el decremento de la humedad del aire (Fig. 5). Se ha observado en algunas especies sobretemperaturas de 10 a $15^{\circ} \mathrm{C}$ dando temperaturas foliares de 46 a $48^{\circ} \mathrm{C}$ (Walter, 1971; Taylor y Sexton, 1972;) sugiriendo que el estres térmico puede ser un factor importante que afecta a las hojas bajo radiaciones moderadas y altas. Bajo humedades altas y bajas radiaciones, la temperatura foliar es muy parecida a la del aire.

La frecuencia de estreses térmicos es una función de la variación temporal de la temperatura, de la humedad del aire, y de la radiación solar. En el sotobosque, la temperatura es muy estable (Fig. 2), pero el nivel de radiación y humedad fluctuan ampliamente, y como el microclima resulta de la interacción de la vegetación y el macroclima, las más altas temperaturas y las más bajas humedades del aire coinciden generalmente con las cargas máximas de radiación solar (Fig. 2).

En la Fig. 6 se presenta la transpiración de Coccoloba liebmannii en función de la hora del día y del mes del año. El patrón de variación es muy parecido para las dos fechas ya que en enero (Fig. 6a.) la disminución de la transpiración no es tan marcada como en abril (Fig. 6b). Las velocidades máximas de transpiración observadas en las dos fechas son aproximadamente a las 1000 hora local con .3 y $.6 \mathrm{~g} \mathrm{~m}^{-2} \mathrm{~s}^{-1}$, respectivamente.

Estas diferencias en las velocidades de transpiración se deben principalmente a la disponibilidad de agua, ya que en enero (principio de la época seca) el suelo está más húmedo que en abril (mitad de la época seca).

b) La evapotranspiración.

Estrictamente la evapotranspiración es un factor biofísico menos micro comparado con la transpiración foliar.

La evapotranspiración de un ecosistema natural o cultivado, se puede considerar como la suma de la transpiración de los individuos que conforman al sistema y la evaporación del suelo. La estimación de la pérdida de agua por el dosel vegetal se puede llevar a cabo a partir de modelos biofísicos tales como el balance de energía y el modelo de Penman-Monteith.

La evapotranspiración de todo el sistema vegetal se puede determinar a partir del balance de energía dado a continuación:

$$
\mathrm{Rn}=\mathrm{LE}+\mathrm{H}
$$

donde $\mathrm{Rn}$ denota la radiación neta o disponible, $\mathrm{L}$ es el calor latente de vaporización del agua, LE es el flujo de calor latente y $\mathrm{H}$ es el calor sensible. La ecuación 2 establece que la energía disponible se conserva como calor latente y sensible.

Introduciendo a la ecuación 2 la razón de Bowen $(B=H / L E$ y despejando a LE se tiene que: 


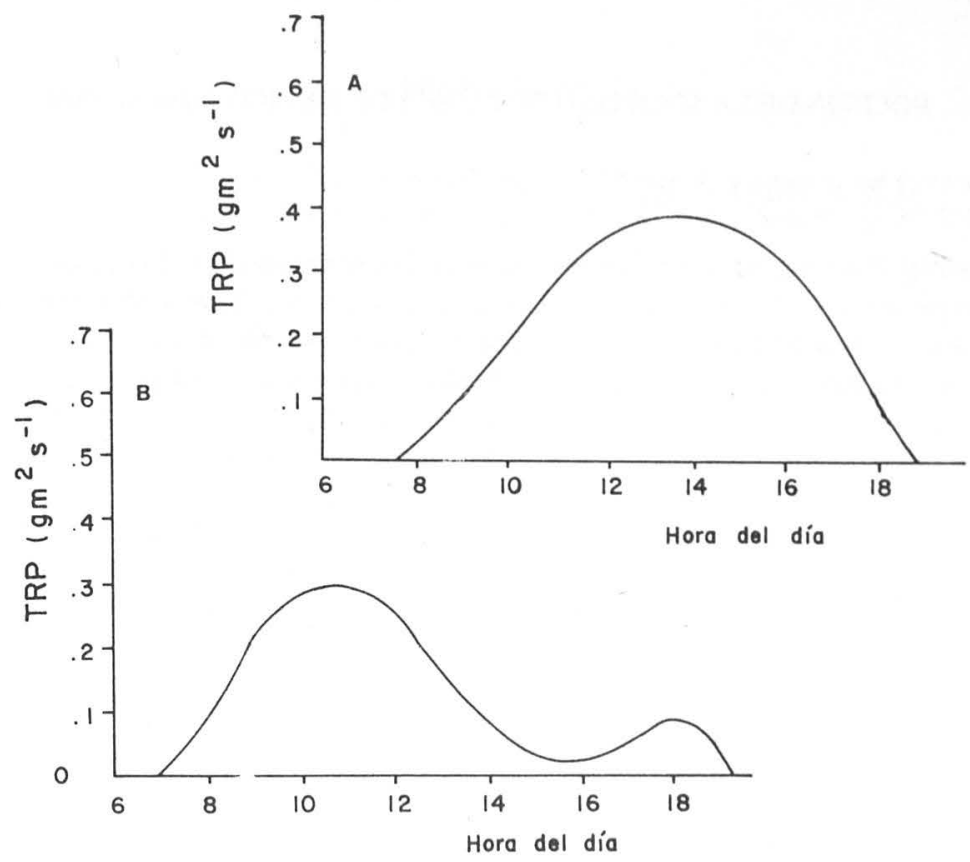

Fig. 6. Transpiración TRP en Coccoloba liebmmanii en una selva baja caducifolia de la costa de Jalisco (según Fanjul y Barradas, 1985).

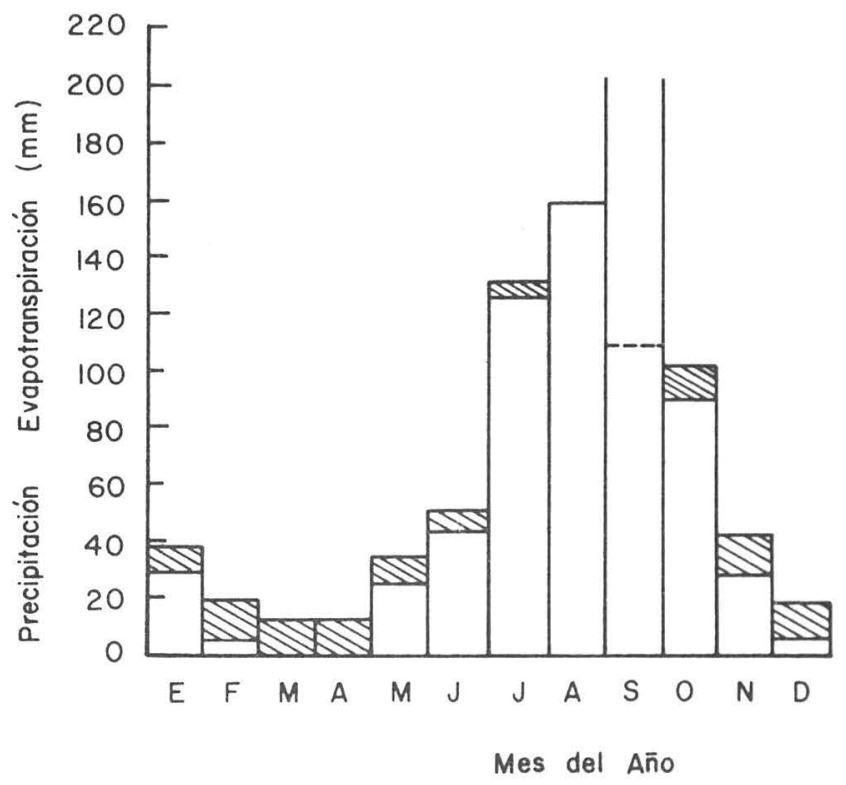

Fig. 7. Balance hídrico de una selva baja caducifolia de la costa de Jalisco (Según Barradas y Fanjul, 1985). 


$$
\mathrm{LE}=\mathrm{Rn} /(1+\mathrm{B})
$$

En la Fig. 7 se presenta un balance hídrico en función de la precipitación y de la evapotranspiración (ET) estimada a partir de la ecuación 3. Este balance se realizó en una selva baja caducifolia de Chamela, Jalisco. Como puede observarse marzo y abril fueron los meses más secos $(\mathrm{Pp}=0)$, y tambien con menor evapotranspiración $(12 \mathrm{~mm})$ mientras que julio, agosto y septiembre son los más húmedos, con 123, 160, $218 \mathrm{~mm}$, respectivamente. Las áreas achuradas de la gráfica representan el déficit hídrico, puesto que ET excedió a la precipitación.

Es importante hacer notar que, si bien agosto presentó una alta precipitación, también muestra una alta demanda evapotranspirativa, de tal forma que no hubo almacenamiento. La recarga hídrica del sistema se llevó a cabo en septiembre, en donde ET alcanzó $110 \mathrm{~mm}$, lo que equivale aproximadamente al 50\% de la precipitación total de ese mes.

\section{CONCLUSIONES Y ESTUDIOS FUTUROS}

Existen numerosos estudios del microclima a nivel mundial, tanto en cultivos como en ecosistemas naturales; sin embargo, en México, la micrometeorología comienza a desarrollarse. Al comparar estos resultados micrometeorológicos con los obtenidos en otras partes del mundo (Aoki et al. 1975), se encontró que los gradientes son muy similares, pero entre estratos, estos ecosistemas parecen ser diferentes. La variable que difiere más es la humedad y tambien es la que presenta mayores dificultades para su medición, lo que sugiere que es sumamente necesario enfocar la atención en el problema de medir la humedad de una manera sencilla y barata.

Los gradientes de humedad y las diferencias de la misma entre sitios tiene importantes implicaciones en la transpiración; debido a que la radiación solar y la temperatura del aire son más altas por encima del dosel vegetal, generando una humedad baja y con ello una alta transpiración (si la conductividad estomática es alta) o una alta temperatura foliar (si la conductividad estomática es baja). Considerablemente, se necesita realizar estudios más enfatizados a las propiedades estomáticas en las selvas y las consecuencias fisiológicas de las altas temperaturas foliares.

En general, la transpiración en la zona intertropical no es muy alta (Landsberg, 1984), pero no se conocen las razones del uso del agua por la vegetación tropical y se necesita mucho más información sobre los balances energéticas, comportamiento estomático y perfiles microclimáticos.

\section{LITERATURA CITADA}

AOKI, M. K.,YABUKI y H. KOYAMA. 1975. Micrometeorology of primary production of a tropical rain forest. J. Agric. Meteor. (Tokio) 31: 115-124. 


\section{MICROCLIMA}

BARRADAS, V.L. y L. FANJUL. 1986. Microclimatic characterization of shaded and open-grown coffee (Coffea arabica L.) plantations in Mexico. Agricultural and Forest Meteorology 38: 101-112. 1985. Equilibrio hídrico y evapotranspiración en una selva tropical caducifolia de la costa de Jalisco México. Biótica 10: 199-211.

1983. Estudio micrometeorológico de la radiación solar en cafetales a la sombra. Simposio Interno del Proyecto "Manejo Integral del Bosque Mesófilo". Instituto Nacional de Investigaciones sobre Recursos Bióticos. Memorias publicadas No. 8330150.

GATES, DM. 1980. Biophyisical Ecology. Springer-Verlag. New York, Heildelberg, Berlín.

FANJUL, L. y V. L. BARRADAS. 1985. Stomatal behaviour of two heliophile understory species of a tropical deciduous forest in Mexico. J. Appl. Ecol. 22: 943-954.

JARVIS, P. G. Y J. I. L. MORISON. 1981. The control of transpiration and photosynthesis by stomata. pp. 247-279. En: Jarvis, P. G. y T. A. Mansfield (Edrs.) Stomatal phsiology. Cambridge University Press, London.

LANDSBERG, J.J. 1984. Physical aspects of the water regime of wet tropical vegetation. pp. 13-26. Physiological Ecology of Plants in the Wet Tropics (E. Medina, H.A. Mooney y C. Vázquez-Yanes Edrs.), Dr. W. Junk Publishers. The Hague, Boston.

TAYLOR, S.E. y O. J. SEXTON. 1972. Some implications of leaf tearing in the Musaceae. Ecology 53: 143-149.

WALTER H. 1971. Ecology of tropical and subtropical vegetation. Edinburgh, Oliver and Boyd Press. 